\title{
Correction to: The First Brazilian Thesis of Evolution: Haeckel's Recapitulation Theory and Its Relations with the Idea of Progress
}

\section{Ricardo Francisco Waizbort ${ }^{1}$ (D) - Maurício Roberto Motta Pinto da Luz ${ }^{1}$ (D) . Flavio Coelho Edler ${ }^{3} \cdot$ Helio Ricardo da Silva ${ }^{2}[$}

Published online: 1 November 2021

(c) Springer Nature B.V. 2021

\section{Correction to: Journal of the History of Biology https://doi.org/10.1007/s10739-021-09651-8}

In this article the affiliation details for Authors Ricardo Francisco Waizbort and Maurício Roberto Motta Pinto da Luz were incorrectly given as 'Servidor em Saúde Pública, Casa de Oswaldo Cruz/Fundação Oswaldo Cruz, Rio de Janeiro, RJ, Brazil' but should have been 'Laboratório de Avaliação em Ensino e Filosofia das Biociências, Instituto Oswaldo Cruz/Fundação Oswaldo Cruz, Rio de Janeiro, RJ, Brazil’.

The original article has been corrected.

Publisher's Note Springer Nature remains neutral with regard to jurisdictional claims in published maps and institutional affiliations.

The original article can be found online at https://doi.org/10.1007/s10739-021-09651-8.

Ricardo Francisco Waizbort ricw@ioc.fiocruz.br

Maurício Roberto Motta Pinto da Luz mauluz@ioc.focruz.br

Flavio Coelho Edler edler@focruz.br

Helio Ricardo da Silva heliorsilva@gmail.com

1 Laboratório de Avaliação em Ensino e Filosofia das Biociências, Instituto Oswaldo Cruz/ Fundação Oswaldo Cruz, Rio de Janeiro, RJ, Brazil

2 Departamento de Biologia Animal, Instituto de Ciências Biológicas e da Saúde, Universidade Federal Rural do Rio de Janeiro, Rio de Janeiro, Brazil

3 Servidor em Saúde Pública, Casa de Oswaldo Cruz/Fundação Oswaldo Cruz, Rio de Janeiro, RJ, Brazil 